\title{
Devenir histórico de enseñanza de la lectura y la escritura en Colombia*
}

\author{
Historical development of teaching reading and \\ writing in Colombia
}

\section{Desenvolvimento histórico de ensino da leitura $\epsilon$ da escrita na Colômbia}

\author{
Henry Cáceres Gómez \\ Oscar Alberto Firacative Velasco \\ Laura Viviana Parada Rodríguez \\ Jhemmy Astridt Muzuzú Torres \\ Wilmar Geovanny Cortés Hurtado \\ Katherine Ramírez Acevedo \\ July Andrea Morales Rubiano \\ José Enrique Copete
}

Universidad San Buenaventura, Bogotá, Colombia**

* El artículo de reflexión es producto del proyecto de investigación realizado dentro de la especialización en Didácticas de las lecturas y escrituras con énfasis en Literatura de la Universidad San Buenaventura, Bogotá, ejecutado desde junio del 2013 hasta mayo del 2014. Grupo de investigación de la especialización de Didácticas de las lecturas y escrituras con énfasis en Literatura.

** Henry Cáceres Gómez, Lic. en Educación Básica con énfasis en Humanidades y Lengua castellana, docente del Colegio Jonathan Swift, Bogotá, Colombia, jerryuniminuto@ hotmail.com; Oscar Alberto Firacative Velasco, Lic. en Educación Básica con énfasis en Humanidades y Lengua castellana, docente del Colegio Jonathan Swift, Bogotá, Colombia, oscarfiracative@hotmail.com; Laura Viviana Parada Rodríguez, Lic. en Preescolar, Asociación de profesionales de Colombia, Bogotá, Colombia, lauravipr@ hotmail.com; Jhemmy Astridt Muzuzú Torres, Lic. En Español, vinculado a la Universidad Juan N. Corpas, Dpto. de Gestión Humana, Bogotá, Colombia, jezmut2004@hotmail. com; Wilmar Geovanny Cortes Hurtado, Lic. en Lengua Castellana Inglés y Francés, docente del Instituto Henao y Arrubla, Bogotá, Colombia, geovannycortes@gmail. com; Katherine Ramírez Acevedo, Lic. en educación preescolar, Bogotá, Colombia, dolphin_kathe@hotmail.com; July Andrea Morales Rubiano, Lic. en educación preescolar, Bogotá, Colombia, andremr_63@hotmail.com; José Enrique Copete, Comunicador social - periodista, docente del Colegio Colombo Británico, Cali, Colombia, jecopete@yahoo. es. El proyecto de investigación fue dirigido por Alba Yaneth Cabra Gómez, Magister en Ciencias de la Educación, Universidad San Buenaventura, Bogotá, yaneth138@yahoo.es 
RECIBIDO: 31 DE MARZO DE 2014・APROBADO: 20 DE JUNIO DE 2014

Para citar este artículo: Cáceres, H., Firacative, O., Muzurú,

J., Cortés, W., Ramírez, K., Morales, J. y Copete, J. (2014).

Devenir histórico de enseñanza de la lectura y la escritura en Colombia. Itinerario Educativo, XXVIII (63), 179-194

Resumen. Los lectores y escritores en Colombia son cada vez menos, la tecnología y la inmediatez unida a los intereses generados por los estudiantes, no permiten establecer procesos críticos que conciban resultados que puedan ayudar a favorecer estas falencias. El presente artículo tiene como propósito realizar un recorrido arqueológico de la lectura y escritura en nuestro país, teniendo en cuenta la importancia que exhiben las TIC en la actualidad frente a estos procedimientos, no con el fin de dar soluciones, sino con la intención de generar inquietudes a todos aquellos que piensan que la lectura y la escritura más que una obligación, es un gusto que se ha perdido con el pasar de los años.

Palabras clave. Lectura, escritura, arqueología y tecnología (Tesauro Unesco).

Abstract. Readers and writers in Colombia are increasingly less, technology and immediacy that coupled with interest from the students; do not allow establishing critical processes that develop results that can help promote these flaws. This article has as purpose to do an archeological tour about reading and writing in our country, taking into account the importance which exhibit ITC today against these procedures, not in order to provide solutions, because the intension to generate concerns to everybody who think that reading and writing rather than an obligation it is a taste that it has lost with the passing of the years.

Keywords. Reading, writing, archeology and technology (Unesco Thesaurus).

Resumo. Os leitores e escritores na Colômbia são cada vez mais, a tecnologia eo imediatismo ligado ao interesse gerado pelos alunos, não permitem que os processos críticos concebam resultados que podem ajudar a promover essas deficiências. Este trabalho tem como objetivo realizar uma viagem arqueológica de leitura e escrita em nosso país, tendo em conta a importância da exposição TIC atualmente atender a esses procedimentos, para não oferecer soluções, mas com a intenção 
de gerar diz respeito a todos aqueles que pensam que a leitura e escrita e não uma obrigação é um gosto que se perde com o passar dos anos.

Palavras-chave. Leitura, escrita, arqueologia e tecnologia (Unesco Thesaurus).

\section{Introducción}

La vida profesional del maestro se ve influenciada positivamente por las directrices investigativas hasta ahora recibidas en la especialización, pues cada maestro dentro de su quehacer pedagógico, empieza a observar desde otro punto de vista estos cambios en materia lecto-escritora, los analiza y los pone sobre la mesa para que sean objeto de estudio y los valora frente a los nuevos avances tecnológicos.

La naturaleza de esta investigación surge por el reconocimiento del impacto que ha tenido el sistema educativo con la incursión de las tecnologías de la información y la comunicación en las prácticas pedagógicas en Colombia, principalmente aquellos cambios en la manera de concebir la lectura y la escritura. Estos elementos han tenido una gran importancia en la sociedad, debido que el sujeto se transporta a otro mundo, donde la magia y la imaginación van de la mano y son al mismo tiempo herramientas del aprendizaje. Es entonces como las letras y su interpretación a través de la lectura, juegan un papel vital en la construcción del mundo.

La investigación adquiere un valor académico y social sumamente importante, pues la era tecnológica ha producido un sin fin de cambios en los niños y jóvenes, y en la forma en como ellos aprenden, comprenden y se relacionan entre sí. Es por ello, que el método arqueológico permite rastrear fuentes que dan a conocer la historicidad de esta serie de cambios, para estudiarlos en un contexto actual.

El trabajo de investigación se ha dividido en varios momentos, que han permitido realizar un rastreo de las distintas etapas del desarrollo de la lectura y la escritura, tomando como referentes: La ley 115, los Lineamientos Curriculares de Lengua Castellana 1996, los Estándares Básicos de Competencias en el Lenguaje (MEN, 2006) hasta la Sociedad de la comunicación y la información; transversalmente han estudiado los diferentes planes de lectura y escritura que se han propuesto en el país en los últimos años. 


\section{El método arqueológico como herramienta de investigación}

La metodología de la investigación se desarrolló a través del método arqueológico, "esta estrategia no implica buscar las verdades del pasado sino el pasado de nuestras verdades» (Murillo, 1996, p. 39). Por consiguiente no se pretende dar importancia al pie de la letra a la información de los resultados en la historia, sino a las circunstancias en las que esos resultados aparecieron.

De esta manera, el método arqueológico se centra en las causas que pudieron generar dichas consecuencias. Es decir, no examina la esencia del conocimiento por medio de artes históricas que cumplen con la labor de dejar unos antecedentes de lo ocurrido, sino en analizar los diferentes contextos. El propósito es realizar un recorrido histórico de la concepción de la lectura y la escritura en Colombia, a partir del uso de la tecnología en la formación del sujeto, en un periodo comprendido entre los años 90 hasta la sociedad de la comunicación y la información. Específicamente hasta el año 2008, en el que se plantea una política pública que da cuenta de proyectos e infraestructura que buscan erradicar para el 2016, el analfabetismo en zonas rurales y urbanas. Además, plantea un fuerte compromiso con la alfabetización y los espacios de lectura para generar un hábito lector que propicie un desenvolvimiento oportuno en este aspecto, para tal fin se pondrá a disposición materiales didácticos (libros) en lengua materna e inglés.

En el desarrollo de la investigación se tiene en cuenta elementos que permiten desarrollar el método arqueológico, como: el archivo que se define como un sistema de enunciados y cosas, pues mediante la construcción histórica se pueden analizar diferentes discursos que permiten exhortar datos claves dentro de la excavación, gracias a ellos se han podido construir enunciados que muestran la realidad lectora y escritora en Colombia con todo su devenir histórico; el enunciado implica observar y releer, para lograr una reflexión de los elementos en cuestión, es por esto, que la apuesta en este sentido, se centra en esos puntos de acuerdo y desacuerdo que emanan de los enunciados lectura y escritura para enriquecer la investigación, Foucault (1985) menciona que:

El enunciado puede ser actualizado en diversas prácticas discursivas de la lectura y la escritura, prácticas de determinación e iluminación 
de un dominio que son interpeladas por las modalidades discursivas, la formación de los objetos, la formación de las modalidades enunciativas, etc. En este contexto, las unidades discursivas no deben buscarse en la coherencia de los contextos, sino del lado de su emergencia simultánea y sucesiva, de desviación de la entidad que los separa y eventualmente de su compatibilidad (p. 3).

Otro elemento es la tematización que se define como la búsqueda arqueológica que se hace para tener una base teórica fuerte y que respalde los planteamientos puestos en la investigación. Es por esto que su importancia en el proceso es vital para guiar por buen camino el proyecto a desarrollar. Según Zuluaga (1999) la tematización es una descomposición o una desarticulación de los registros. No es etimológica, gramatical, lógica, lingüística o de los actos de habla; en su lugar, se pretende descomponer desde una lógica relacional. Es por ello, que las relaciones se determinan por las funciones que cumplen en los documentos cada uno de los componentes que se identifican como nociones, conceptos, objetos.

Mientras que las rejillas de especificación según Foucault (1999) son los sistemas según los cuales se separa, se opone, se entronca, se reagrupa y se clasifica los diferentes objetos, en este caso la lectura, la escritura y la tecnología. En este campo el empleo de la rejilla de especificación permite emplear estos elementos, a partir de lo que en la investigación es relevante, los cuales están articulados en los enunciados de lectura, escritura y tecnología, dentro de un tiempo determinado.

Gráfico 1. Rejilla de especificación

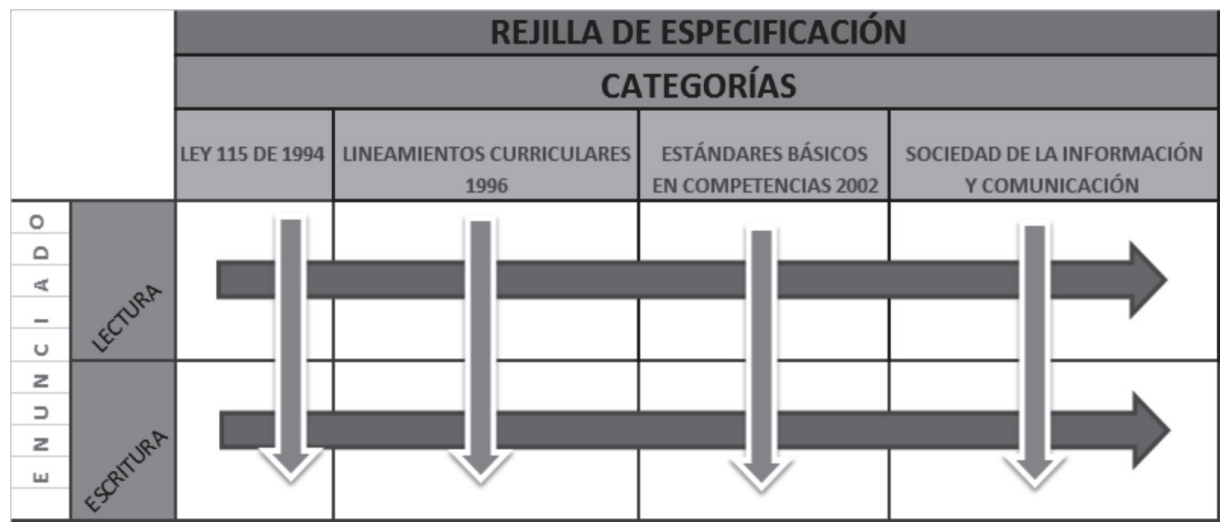

ITINERARIO EDUCATIVO • ISSN OILI-2753 • AÑO XXVIII, N. ${ }^{\circ} 63$ • ENERO - JUNIO DE 2OI4 • P. 179 - 194 
La categoría, de acuerdo con Gomes (2003): “La palaba categoría, en general, se refiere a un concepto que abarca elementos o aspectos con características comunes o que se relacionan entre sí. Esa palabra está relacionada a la idea de clase o serie" (p. 55). Es por ello, que en cualquier tipo de investigación se requiere establecer clasificaciones, lo cual implica agrupar elementos, ideas o expresiones que tengan relación con un concepto que abarque lo anterior. En este sentido, el investigador debe hacer una formulación de sus categorías al iniciar el proceso de investigación pero, igualmente, debe estar en capacidad de reformularlas o complementarlas de acuerdo con las evidencias y a los resultados que vaya obteniendo en las diferentes etapas del mismo.

Así pues, el método arqueológico brinda los elementos necesarios para desarrollar la construcción investigativa a partir de una realidad, en un tiempo, lugar o contextos determinados, permite indagar las condiciones por las cuales los estudiantes en la actualidad se acercan a la lectura y la escritura en una sociedad enmarcada por la comunicación e información, bajo intereses diferentes a los de otros tiempos.

\section{Del cáncer de lo canónico a lo consumista}

La lectura constituye más que una interpretación de fonemas, en palabras del escritor colombiano Ospina (2003) es mucho más de lo que nos enseña la alfabetización, es mucho más que organizar las sílabas y reconocer las palabras, es un acto creador sutil y excitante, es una fuente de información, de conocimiento y de sabiduría; es también una manía, una obsesión, un tranquilizante, una distracción y sobre todo una felicidad. Siendo así y teniendo en cuenta el sentido pragmático de la lectura en la educación colombiana, se puede hacer un recorrido histórico que permite vislumbrar cómo se ha llegado a un punto en el que la lectura ya no se concibe igual.

La estudio realizado en este proyecto investigativo muestra eventos claves en la historia de la educación en Colombia, la cual después de haber vivido momentos históricos bajo el yugo de una colonización e imposición de fuertes modelos europeos, debe aprender a leer de acuerdo a los dogmas de la iglesia católica y como lo afirma Patiño (1995) no tiene en cuenta al individuo sino a las directrices y objetivos marcados por esta institución religiosa. 
A pesar de haber vivido una educación rígida, que no daba al sujeto un momento para la crítica, se toman influencias de la escuela activa, encabezada por Decroly y Monchap (1983) en donde se tienen en cuenta elementos nunca antes considerados como los intereses particulares del sujeto o las formas en las que este debe aprender y en palabras de Nieto (1924) "Se acaban los siervos y aparecen los investigadores". Con estos antecedentes y la inquietud de conocer la posición legislativa colombiana en cuanto a la lectura y el devenir, se empieza a hacer un recorrido histórico desde los años 90 hasta la sociedad de la información y la comunicación.

En 1991 y con una realidad enmarcada por un contexto violento y quizá con el inicio de la era del narcotráfico, donde se muestran modelos de vida diferentes y que marcarían trascendentalmente la forma de ver el mundo por los niños y nuevas generaciones, es allí con la expedición de la Ley 115 cuando se tienen en cuenta por primera vez en la historia nacional, según Rincón (2003): "la interpretación y comprensión como condiciones imprescindibles en todo acto de lectura" (p. 72).

Con la creación de Fundalectura en 1990 y los proyectos de la Secretaría de Educación se busca hacer un país de lectores, que involucren la ciencia y la tecnología con el acto de leer. Es así, como se incursiona en la sociedad de la información y la comunicación, usando herramientas que son atrayentes para los nuevos lectores. Además, se busca garantizar el acceso a la cultura escrita como vía de equidad e inclusión social y de desarrollo de la ciudadanía, con el Plan Nacional de Lectura y Escritura (PNLE) del 2010, cuyo objetivo principal fue:

Fomentar el desarrollo de las competencias comunicativas, a través del mejoramiento del comportamiento lector, la comprensión lectora y la producción textual de estudiantes de educación preescolar, básica y media, fortaleciendo la escuela como espacio fundamental para la formación de lectores y escritores y vinculando la familia en estos procesos (s.p.).

Proyectos altamente interesantes que han tenido un alto impacto social, pero puede decirse que no tanto como el que se espera, pues es aún cuando los desempeños en lenguaje muestran la misma cultura literaria que se limita a lo literal, carente de sentido crítico y que a pesar de tener herramientas cada vez más accesibles para cualquier tipo de comunidad, se alejan del mundo letrado.

ITINERARIO EDUCATIVO • ISSN OIDI-2753 • AÑO XXVIII, N. ${ }^{\circ} 63$ • ENERO - JUNIO DE 2OI4 • P. I79 - I94 
Según encuestas realizadas por el DANE en los mejores años, el promedio de libros leídos por habitante no llega a los dos, sin embargo, algo significativo ocurre en la encuesta del 2005 y que se hace evidente en las cifras, después de un lustro, es el crecimiento de la lectura en internet, lo cual no es una sorpresa pues desde la encuesta del año 2000 el fenómeno empezaba a hacerse evidente en la accesibilidad al servicio y la expansión tecnológica.

Esta expansión habla del incremento de la "lectura tecnológica" y la reflexión que realiza Cassany (2006): “Es mucho más fácil crear una tecnología que cambiar los hábitos de las personas que tenemos que usarla" (p. 57), y explica que aunque la literacidad electrónica este a la orden del día, es muy complicado renovar los procesos de pensamiento, cambiar hábitos, perder el miedo a lo nuevo y por ende desconocido.

Pero ello en lugar de despejar el panorama, abre puertas a nuevos interrogantes ¿Cuál es la calidad de la lectura en la actualidad? ¿Es y seguirá siendo Colombia un país de lectores influenciables por la moda de la época? ¿Tiene el colombiano una postura crítica frente a lo que lee?

\section{Escritura en contexto}

La lengua escrita está presente en la interacción del ser humano con sus pares y los elementos que lo rodean. Su uso se da de forma natural y va creciendo por las prácticas individuales y sociales en los diferentes contextos del hombre. Desde el ejercicio pedagógico se ha buscado proveer a los estudiantes las herramientas necesarias para dominar el acto de escribir, respondiendo a las características sociales que se transforman con el paso del tiempo.

Partiendo de esa necesidad, ingresan al aula ejercicios de aprestamiento, métodos globales y sintéticos, más conocidos como métodos tradicionales que tienen como finalidad la formación de escritores. Sin embargo, al analizar más de cerca estos métodos, se evidencia que el acto escritor carece de significado debido a que el estudiante es visto como un sujeto sin conocimiento escritor y sin habilidades desarrolladas para adquirir algún tipo de escritura.

De acuerdo con lo anterior se empieza a plantear la necesidad de reconocer las habilidades que el niño desarrolla en su entorno mucho antes de llegar al aula. Al respecto Villegas (1996) menciona: 
El niño que ha llegado a la escuela ha internalizado un sistema de reglas pragmáticas para la enunciación cotidiana. El conocimiento de su lengua lo ha construido en el curso de un proceso de organización mental que supone la capacidad de producir estructuras profundas y superficiales, relacionándolas entre sí (p. 50).

Así entonces, es necesario incluir en el proceso de enseñanza de la escritura el contexto y las experiencias de los estudiantes, de tal manera que este aprendizaje sea significativo para cada uno de ellos.

La contextualización de este proceso pretende lograr el aprendizaje de la lengua escrita empleando la interacción presente entre leer, hablar y escribir como medio para la creación de conocimiento, el uso de recursos narrativos, la interpretación y la comunicación de mensajes en un texto. Es importante reconocer que no solo en el aula se desarrollan procesos escritores sino que las experiencias presentes en el entorno deben convertirse en posibilitadoras del acto escritor.

\section{Las Tecnologías de la Información y la Comunicación (TIC) un mundo para innovar}

Las TIC, desde su llegada al mundo, comenzaron a especular sobre los posibles impactos que esta podría tener en la educación y en el desarrollo de un país, abriendo puertas al conocimiento de nuevas generaciones, logrando cambios significativos en la sociedad forjando una rebelión en la educación.

Por consiguiente, las TIC dejaron de ser una curiosidad sobre la tecnología, para transformarse en una gran herramienta en el desarrollo social y económico de todos los países, en especial los que se encuentran en vía de desarrollo, de ahí que la generación de nuevos empleos se encuentran relacionados con la inclusión de la misma con el fin de lograr la competitividad del país y por consiguiente su desarrollo en un mundo globalizado.

La imparable y competitiva expansión de los medios de comunicación provoca una búsqueda permanente del lenguaje llano que pueda llegar a todas las audiencias potenciales, tratando los temas actuales que interesan y recogiendo la creatividad y los usos lingüísticos de la calle. Periódicos, radios y televisiones se afanan por elaborar un estilo expresivo propio y adecuado a los tiempos modernos (Cassany, 1993, p. 14). 
Por consiguiente los progresos de la sociedad están soportados en los medios electrónicos, es por ello que ante la necesidad del manejo de la información industrializada y tecnificada en la vida diaria, se vio la necesidad de involucrar la lectura y escritura a estos medios, comprendiéndolos como el producto de la transición de los procesos escriturales a la alfabetización, traducida en la concepción, de que el desarrollo social de los países va de la mano de un buen proceso de asimilación con las letras.

No se puede negar que la llegada de internet concluyó un cambio en la manera de leer y escribir, puesto que la información que producía la misma permitía un acceso al conocimiento de una manera sencilla y organizada para los usuarios optimizando tiempo y recursos. En Colombia oficialmente el internet llegó en 1995, no obstante hubo un agravante, solo la clase alta podía acceder a este servicio que en ese entonces se consideraba costoso y una ostentosa herramienta que no todos podía tener.

El uso de una tecnología como Internet no es la relación con un objeto, sino con el universo de representaciones culturales con las cuales esa tecnología se articula en la vida social de los jóvenes. Internet es un objeto que se apropia en un universo relacional donde otros objetos, espacios y prácticas lo 'resignifican'. Pensar el impacto de Internet en los mundos de vida de los jóvenes es indagar por la estructura de significados en donde Internet se inscribe y por el nudo de relaciones que con él se establece... De esta forma, asumimos que lo que ocurre con Internet está en relación tanto con el uso del objeto como en los significados con los cuales se lo representa (Bonilla y Cliche, 2001, p. 43).

A lo anterior se agrega el gran cuestionamiento de si la educación ha cambiado con los años, o es la misma forma de enseñar a leer la de hoy a épocas atrás, será tal vez que el cambio generado fue de la sociedad y no de las formas de leer y escribir. "Es urgente desarrollar la lectura como una competencia que funda y atraviesa cualquier carrera y que, a la vez, posibilita tanto el desarrollo personal como colectivo" (Vásquez, 2011, p. 196).

Por ende la enseñanza de la lectura se ha transformado por las TIC ya que pueden ser herramientas que permiten identificar la manera de leer de los jóvenes y que leen, como incorporan a través de ella nuevas culturas en su contexto diario, desde la interacción social entre pares 
hasta el aula de clase, lo cual presenta un nuevo sentido, de este modo se socaban la ignorancia y se abren áreas de discusión, concordancia o divergencia con otras opiniones, ampliando el pensamiento.

Por esta razón, es necesario enseñar habilidades para el correcto manejo de las tecnologías, entendiendo que cada individuo posee unos conocimientos previos de las mismas, estas pueden ayudar más que perjudicar, debido a que ofrecen diferentes perspectivas, los conecta a nuevos avances tecnológicos mediante, debates, charlas, investigaciones un sinfín de información que es fácil hallar en diferentes medios de comunicación, que desarrollan un pensamiento científico y crítico.

Es evidente que falta mucho por recorrer con las nuevas tecnologías, ciertos obstáculos los cuales se deben trabajar mancomunadamente fortaleciendo en nuestros niños, niñas y jóvenes proyectos investigativos encaminados a fortalecer el desarrollo intelectual, crítico, y analítico. No olvidemos que la lectura y escritura tradicional hoy día están perdiendo su valor, quien no domina los medios electrónicos no es competente en la sociedad actual.

De esta manera, se ha llegado al punto de entender la importancia de las TIC que lo han abarcado todo, pero con el difícil escenario de una escuela enfrentada a un mundo tecnológico, que rutinariamente pone a prueba las prácticas que se realizan por parte de los sujetos que proponen la enseñanza en la comunidad educativa.

Para finalizar se podría afirmar que muchos son los retos que plantean las nuevas tecnologías frente al propósito de enfocar la lectura y la escritura, pero está claro que las soluciones no llegaran de la nada o de seudocambios generados por el sistema, especialmente el colombiano, esto depende de docentes que investiguen con el fin de crear conciencia tanto en colegas, como en la población en general, crear pequeñas transformaciones que formen cadenas tan fuertes donde su eslabón más débil resista todas las embestidas que genera el desgaste de la renovación del tiempo.

\section{Hallazgos arqueológicos}

Las nuevas tecnologías de la información y la comunicación, consideradas como instancias mediadoras entre los sujetos y la realidad, pueden en muchos casos propender por la desintegración, ya que influyen para que 
se desarrollen comportamientos que fomentan la distancia. Un ejemplo de esto es la individualidad que se propicia cuando se establece una relación de dependencia con estas.

Estos medios se reconocen como elementos de lo posible, ya que motivan encuentros y desencuentros, generando en muchos casos apegos. Este el reto que tienen otras actividades constructoras de conocimiento como la lectura, en donde se hace una búsqueda a través de las grafías que permite el reconocimiento y encuentro consigo mismo y con los demás.

Es por eso que el texto, el contexto y el lector, son una verdadera comunidad tripartita, permitiendo una verdadera función dialogante. Es la lectura un instrumento que permite la interpretación, para así acceder a la construcción y reconstrucción de mundos posibles. Pese a que los individuos alejados de la vida, del mundo, del cambio generado por las grandes revoluciones universales, han fundado desapegos por el entorno; situación evidente el ser humano, quien oculta un bagaje inconmensurable de las modas, estilos, tendencias y aceptaciones. Por eso se puede decir que la escuela termina cediendo a la fuerza de este caudal llamado: superficialidad.

A partir de las revoluciones educativas, las personas toman decisiones diferentes y especiales, dejando de ser un individuo pasivo y recolector de cantidades de información, para erigirse como sujeto activo y cognoscitivo, que considera su entorno como el objeto de conocimiento, al que debe dirigirse a través del contacto.

El maestro participa como orientador y posibilitador de experiencias lectoras entre el sujeto y objeto del conocimiento. Pese a que el profesor y el estudiante están inmersos en una jerarquía, no se debe dejar a un lado la finalidad del ser humano, es decir, la relación con el pensar y el hacer, ya que las estructuras mentales solo se logran a través de la interacción con el entorno.

La dialéctica de la literatura con otras disciplinas permitirá un mejor y profundo conocimiento. El problema actual de la escuela en todos sus niveles, es que muy pocos veces genera posibilidades para que los estudiantes puedan leer desde sus preguntas, desde sus deseos, a cambio de ello les imponen textos y lo más lamentable, "métodos" que por muy "sofisticados y modernos" que puedan ser, en general tienden a castrar el gusto por la lectura, la escritura y la discusión. 
La lectura desarrolla una construcción personal para darle sentido a la realidad vivencial y experimental, por eso no es factible el préstamo de verdades ajenas, ni proporcionar recetas para el aprendizaje de ésta. El conocimiento se debe entender como algo personal, en donde es fundamental el ser y el hacer, que traducidos permiten expresar el mundo.

La lectura debe estar enfocada al logro de la observación, la descripción, la comparación y la clasificación, en donde sea fundamental la adquisición de destrezas, para que quien la realiza logre la justificación y verificación de lo interpretado. Ésta permite una relación dialéctica, fomentando el interés por la búsqueda de la verdad. El espíritu investigativo se da a través del acto lector, ya que desliga al ser humano de un plano estático, para sumergirlo en mundos posibles. Por eso, dicho acto no puede estar dirigido por intereses particulares, lo recomendable es que éste sea un sinónimo de la libertad en un individuo que está inserto en la sociedad.

El acto de leer permite la individualización, el encuentro personal e interpersonal. El ser humano es único y su interpretación está mediada por diferentes factores circunstanciales, no puede ser observado como uno masa. Es por eso que el ejercicio de la lectura facilita el entendimiento, ya que fomenta el respeto por las ideas ajenas, así como por las propias. La educación fomenta la particularidad con el uso correcto de habilidades, para alcanzar el máximo logro que impactara positivamente al entorno social. La lectura reconoce a un individuo integrado por la sociedad, no divorciado de ella.

Los maestros tienen la misión de ser garantes en el proceso lector de sus estudiantes, participando a través de la observación activa, generando estímulos que potencialicen el aprecio por este acto netamente humano. Ésta es una actividad interdisciplinaria, intelectual y reflexiva que humaniza. Es igualmente, un ejercicio semiológico que permite reconocer en cada cosa un elemento suelto, o encadenado con el conocimiento.

La lectura permite descubrir el mundo y la escritura mantiene y desarrolla la cultura. Por eso es importante resaltar que la lectura y escritura trascienden mientras se fundamenten en posiciones estéticas y sensibles.

Son muchas los interrogantes que surgen con referente a los procesos de lectura - escritura que se implementan en la educación de hoy. Es importante reconocer que la tecnología es un gran eslabón que muchos 
maestros están iniciando a investigar para poderlos incluir como herramientas pedagógicas en el aula de clase, sin embargo hay que tener presente que muchas veces no se le están dando el adecuado uso.

En este proceso de cambios en cuanto a leyes educativas y formas de leer, es evidente que además de los innumerables esfuerzos gubernamentales por renovar los proyectos en materia de lectura, se debe trascender en la cultura misma y al sistema de valores, para que permitan un acercamiento al acto de leer.

Para construir sujetos lectores sería preciso estudiar los factores socio - culturales que lo circundan y comprender que en una sociedad de la información y la comunicación se tienen necesidades diferentes a la de los antepasados, es por ello que la revolución educativa debe ser desde el interior del núcleo familiar, para así lograr la abolición de paradigmas lecto-escritores obsoletos que dejan de un lado al ser, sus necesidades y sus nuevos retos.

De esta manera cuando se habla de escritura, suele pensarse en ésta como un proceso de reconocimiento de las letras con la finalidad de escribir algún tipo de texto. Sin embargo, esa mirada es limitante y empobrecedora, debido a que la escritura es un acto comunicativo que no conoce fronteras y que puede encontrar reconocimiento en lectores inesperados.

Sin embargo, se menciona también que este proceso de aprestamiento genera una descontextualización en la escritura y la lectura ya que las reduce a simples habilidades motrices, creación de grafías y lectura de fonemas. Ya que el niño está en un sistema que desarrollara primero su dimensión cognitiva que comunicativa, siendo esta última la que ejecuta el niño en sus primeros años de vida, ya que antes de leer él se comunica verbalmente.

Por lo anterior, se puede llegar a la conclusión que se observa la necesidad de realizar una reforma a la enseñanza aprendizaje de la escritura, donde se tenga en cuenta que el niño antes de llegar al aula tiene unos preconceptos que ha adquirido en su constante intercambio de experiencias con sus pares y sujetos de su entorno, que él ya se comunica, relata pequeños hechos y vivencias, un método no estricto este debe ser flexible a la necesidad de lo que cada niño posee. 
Hacer una reflexión sobre la necesidad de incluir en ese proceso dos aspectos, siendo el primero de ellos el de acercar a los estudiantes a la adquisición de las habilidades propias del acto escritor y, el segundo, orientarlos hacía una escritura desde sus vivencias y su realidad. Es así como cada escritor descubre que no se encuentra solo en la gran aventura de escribir.

Es por esto que las TIC han cambiado la manera de leer y escribir en nuestra sociedad, sin embargo, estas tecnologías no demostraron que las antiguas maneras de hacer las cosas pasaron a la historia, es decir que quedaron obsoletas en su totalidad.

No se pretende afirmar que las TIC estén del todo mal, pero, este factor es un error, si se cree que las tecnologías son la solución a los inconvenientes que se han tenido desde el pasado en nuestro país. La falta de lectura y escritura no es una dificultad actual, es una herencia que se ha alterado desde épocas remotas, así que confundir las soluciones, no es un acierto eficaz para enfrentar el problema en mención.

\section{Referencias}

Bonilla, M. y Cliche, G. (2001). Internet y sociedad en América Latina y el Caribe, investigaciones para sustentar el diálogo. Quito: Flacso.

Cassany, D. (1993). La cocina de la escritura. Barcelona: Editorial Empúries.

Cassany, D. (2006). Tras las líneas: sobre la lectura contemporánea. Barcelona: Anagrama.

Decroly, O, Monchamp, E. (1983) El juego educativo. Iniciación a la actividad intelectual y motriz. Madrid: Morata.

MEN (2006). Estándares Básicos de Competencias del Lenguaje. Bogotá: Ministerio de Educación Nacional.

Foucault, M. (1985). Arqueología del saber. México: Siglo XXI.

Foucault, M. (1999). Arqueología del saber. México: Siglo XXI.

Gomes, R. (2003). Investigación social. Buenos Aires: Lugareditorial S.

Murillo, S. (1996). El Discurso de Foucault: Estado, locura y anormalidad en la construcción del individuo moderno. Buenos Aires: Oficina de publicaciones del CBC.

ITINERARIO EDUCATIVO • ISSN OIDI-2753 • AÑO XXVIII, N. ${ }^{\circ} 63$ • ENERO - JUNIO DE 2OI4 • P. I79 - I94 
Nieto, A. (1924). Sobre el problema de la educación nacional. v. 50. Bogotá: Editorial Minerva.

Ospina, W. (2006). Lo que entregan los libros. Por qué leer y escribir. Bogotá: Alcaldía Mayor de Bogotá.

Patiño, C. (1995). El lenguaje de los afrocolombianos y su estudio. Estudios de literatura y cultura colombianas y de lingüística afro-hispänica. Frankfurt am Main: Editorial Lang.

Plan Nacional de Lectura y Escritura (2010). Bogotá, Colombia: Ministerio de Educación Nacional. Disponible en http://www. mineducacion.gov.co/1621/w3-article-325393.html

Vásquez, R. (2011). Educar con maestría. Bogotá: Editorial CMYK.

Villegas, O. (1996). Escuela y lengua escrita: competencias comunicativas que se actualizan en el aula de clase. Bogotá, Colombia: Cooperativa Editorial Magisterio.

Zuluaga, O. (1999). Pedagogía e Historia: La Historicidad de la Pedagogía, la enseñanza, un objeto de saber. v. 1. Medellín: Editorial Universidad de Antioquia. 